\title{
Time perception with and without a concurrent nontemporal task
}

\author{
NANCY S. HEMMES, BRUCE L. BROWN, and CHRIS N. KLADOPOULOS \\ Queens College, City University of New York, Flushing, New York \\ and The Graduate Center, City University of New York, New York, New York
}

\begin{abstract}
Prospective time estimates were obtained from human subjects for stimulus durations ranging from 2 to $23 \mathrm{sec}$. Presence and absence of a concurrent nontemporal task was manipulated within subjects in three experiments. In addition, location of the task within temporal reproduction trials and psychophysical method were varied between groups in Experiments 2 and 3, respectively. For long-duration stimuli, the results of all three experiments conformed to results in the literature, showing a decrease in perceived duration under concurrent task conditions, in accord with attentional resource allocation models of timing. The effects of task location and psychophysical method on time estimates were also compatible with this analysis. However, psychophysical functions obtained under task conditions were fit well by power functions, an outcome that would not be anticipated on the basis of attention theory. The slopes of the functions under no-task conditions were steeper than those under task conditions. The data support the perceptual hypothesis that different sources of sensory input mediate timing under task and no-task conditions.
\end{abstract}

When human subjects are required to engage in a concurrent task while simultaneously monitoring duration under prospective-timing procedures, their temporal estimates are generally shorter than estimates obtained without a concurrent task. Across many studies, previous authors have reported this effect for a fairly wide range of durations (e.g., Block, 1992; Brown, 1995; Brown, Stubbs, \& West, 1992; Fortin, Rousseau, Bourque, \& Kirouac, 1993; Macar, Grondin, \& Casini, 1994; Sawyer, Meyers, \& Huser, 1994; Zakay, 1998) under estimation, production, and reproduction procedures (for a review, see Brown, 1997). Explanations of this finding often refer to Thomas and Weaver's (1975) attentional model, which posits that temporal estimates are influenced by the sharing of cognitive resources between temporal and nontemporal information processing. According to such resource-allocation models of timing (e.g., Brown, 1997; Macar et al., 1994; Sawyer et al., 1994; Zakay, 1998), temporal estimates are mediated by an in-

A portion of the results of this study was reported in papers presented at the annual meeting of the Eastern Psychological Association, April 1997 in Washington, DC. The research was supported by grants from the PSC-CUNY Research Awards Program of the City University of New York. We thank John Zhu for his development of software with which to control the experimental procedures and Philip Ramsey for his advice regarding statistical procedures. We also thank Patricia D'Ateno, Randi Sarokoff, and Noel Rosa for their contributions to the study. Correspondence concerning this article should be addressed to N. S. Hemmes, Department of Psychology, Queens College-CUNY, Flushing, NY 11367 (e-mail: nancy_hemmes@qc.edu).

Note-This article was accepted by the previous editorial team, headed by Neil Macmillan. ternal clock that accumulates time units (variously referred to as pulses, temporal cues, or subjective time units) under attentional control. The level of attention to timing governs a switch (Lejeune, 1998) or a gate (Block \& Zakay, 1996) that controls the flow of pulses from a pacemaker to an accumulator. Imposition of a concurrent task reduces the attentional resources available to the timer, resulting in suspension of timing by the switch (reduced flow of pulses to the accumulator) each time attention is directed to the task. Because perceived duration is proportional to the number of time units accumulated, the attention model anticipates that estimates under both task and no-task conditions will be proportional to stimulus duration and that the discrepancy between these two conditions will increase as sample duration increases.

There is evidence of a more complex relation between temporal estimates and stimulus duration under task conditions than that implied by the attentional model. Several studies have indicated that performance under concurrent tasks is not confined to shortening, conforming instead to the principles of Vierordt's law (Woodrow, 1951) - underestimation (with respect to veridical time) of longer intervals and overestimation of shorter intervals (Brown, 1995; Brown et al., 1992; Kladopoulos, Hemmes, \& Brown, 1997; Wearden, Denovan, Fakhri, \& Haworth, 1997). Other reports have indicated that time estimates vary as a nonlinear function of duration under task conditions (Brown et al., 1992; Kladopoulos et al., 1997).

An alternative hypothesis of task effects is that different sensory modalities mediate performance under task versus no-task conditions. This notion, which we term the perceptual hypothesis, arises from the observation that when long temporal intervals ( $>1$ or $2 \mathrm{sec})$ are "timed," 
human subjects resort to chronometric counting, unless they are prevented (or discouraged) from doing so (Wallace \& Rabin, 1960; Zakay, 1990). Under those conditions, the discriminandum for temporal judgments is likely to be self-generated count stimuli-typically, chronological numbers starting with "0" or " 1 ." When counting is discouraged by a concurrent task, a different source of sensory input, arising from either an internal clock mechanism or the sensory properties of the task, must be employed. According to the perceptual hypothesis, time judgments under task and no-task conditions will differ because they are based on perception in different sensory domains (e.g., Stevens, 1960, 1975).

Many procedural and parametric differences have distinguished the reported investigations of concurrent task effects, and in relatively few studies have individual subjects been exposed to a range of temporal stimulus durations under task conditions. In the present study, the effects of a concurrent number-reading task under three different psychophysical methods (reproduction, estimation, and production) were investigated in three experiments. In addition, task placement during temporal reproduction trials was manipulated, with placements occurring in the sample phase, the reproduction phase, or both phases. In order to evaluate the attentional and perceptual hypotheses, a range of durations was employed. Each subject was exposed to seven sample durations ranging from 2 to $23 \mathrm{sec}$, under task and no-task conditions. The number-reading task was designed to deter chronometric counting (cf. Frankenhaeuser, 1959); accordingly, it was anticipated that the subjects' judgments would be based on counting under the no-task condition, but not under the task condition. Data from all experimental conditions were subjected to analyses that addressed the following questions. (1) Are temporal estimates under task conditions proportionally lower than those under no-task conditions and, therefore, in accord with an attentional analysis? (2) Are there reliable differences in psychophysical or psychometric parameters between data from task and no-task conditions, thereby supporting the premise of the perceptual hypothesis that different sources of sensory input mediate timing under task and no-task conditions?

The latter question was addressed in two ways. First, the psychophysical functions in each experiment were subjected to log-log transformations to permit estimation of psychophysical parameters. The influence of different sensory domains would imply differences in the slopes of the functions under task and no-task conditions.

Second, the possibility that different perceptual processes mediate temporal judgments under task versus no-task conditions was evaluated with an analysis of functions relating variability of temporal estimates to stimulus magnitude. Killeen and Weiss (1987) have pointed out that errors in timing (variability) may be attributed to more than one measurable source and that the relative contribution of each source may vary with the experimental procedure and/or the behavior of the subjects. Compo- nent analyses based on this reasoning have been used by Killeen and Weiss (1987), Fetterman and Killeen (1990), and Grondin, Meilleur-Wells, and Lachance (1999) to evaluate the effects on timing of explicit counting. A variation of that strategy, termed a slope analysis, has been described by Ivry and Corcos (1993) and Ivry and Hazeltine (1995). Briefly, the logic of the analysis holds that variance under a timing task is composed of variability attributable to the timing process and of variability unrelated to timing, such as task-specific response variability. By definition, only the former would be expected to vary as a function of sample stimulus duration. Therefore, the slope of the function relating variance of temporal estimates to mean estimates for a range of sample durations reflects the duration-dependent source of variability, whereas the intercept of the function is attributable to duration-independent sources. If a common perceptual mechanism governs performance under task and no-task conditions, task-induced variability would produce an increase in intercept, but no change in slope. If slopes differ, separate timing mechanisms will be inferred.

In addition to the preceding questions, the present study addressed the possibility that concurrent task effects may be attributable to internal clock processes other than, or in addition to, accumulation of time units, as has been described above. This analysis was guided by the structural model of human timing that includes pacemaker, switch, accumulator, memory, and decision processes (Treisman, 1963). Gibbon and Church (1990) have described a methodology for determining the effects of independent variables on memory and decision processes. The analysis entails implementation of the peakinterval procedure - a fixed-interval procedure originally used with animal subjects (Catania, 1970; Roberts, 1981). In the present study, the start-stop procedure (described below), a peak-interval procedure adapted for human subjects by Kladopoulos, Brown, Hemmes, and Cabeza de Vaca (1998) was employed. In a previous study, Rakitin et al. (1998) found that there was no effect of chronometric counting on measures generated by a different version of the peak-interval procedure for repeated trials with a 12 -sec sample. The generality of this finding for a range of durations was evaluated in the present study.

\section{EXPERIMENT 1}

In Experiment 1, two groups of subjects were exposed to stimulus durations ranging from 2 to $22.8 \mathrm{sec}$ under the method of reproduction, using the start-stop procedure. Presence of a concurrent number-reading task, intended to prevent the subjects from engaging in chronometric counting, was manipulated in a within-subjects design. In addition, the effect of presentation of numbers, without the number-reading requirement, was evaluated in a group design. For all the subjects, three- to five-digit numbers were displayed on the computer monitor throughout the sample stimulus on $50 \%$ of the trials. 
The subjects in the active group were required to pronounce the numbers out loud as they appeared, whereas those in the passive group were not required to pronounce the numbers. The passive group was included in order to test the possibility that mere presentation of numbers during the sample stimulus would have an effect on temporal judgments. This possibility was anticipated from evidence of a filled-duration illusion, in which temporal estimates are higher when exteroceptive stimulus changes (e.g., clicks, tones, light flashes, or moving visual images) fill a temporal interval (Brown, 1995; Hicks, Miller, Gaes, \& Bierman, 1977; Poynter, 1989; Thomas \& Brown, 1974; Vroon, 1970). Poynter and Homa (1983), who studied the relation between number of sensory events during the target interval and perceived duration, noted that the positive relation consistent with the filled-duration illusion is evident only at shorter intervals. Poynter (1989) proposed that the contribution of exteroceptive events to time perception is important only when target intervals are so short that subjects are not able (or inclined) to invoke timing strategies, such as chronometric counting. The preceding analysis anticipates that overestimates (at least at short sample durations) will occur for both groups when numbers are presented, but not in their absence.

\section{Method}

\section{Subjects}

Twenty undergraduate psychology students (6 males and 14 females) participated to satisfy an academic requirement. Their ages ranged from 17 to 41 years. The members of each consecutive pair of students who appeared for the study were randomly assigned to one of two groups, active and passive. All the subjects completed each of the experimental sessions. The subjects in this and subsequent experiments were asked to place any timepieces out of sight.

\section{Apparatus}

A Gateway microcomputer (hardware clock rate $=18.2$ ticks $/ \mathrm{sec}$ ) and a VGA color monitor were used to run the experimental sessions and to acquire data. The left button of a Microsoft PS/2 mouse, covered by black electrical tape, served as the manipulandum. The subjects were seated facing the computer screen, with the mouse located directly in front of the computer monitor.

\section{Procedure}

The start-stop procedure. All the trials were conducted under the method of reproduction, using the start-stop procedure. The following sequence of stimuli was presented at the approximate center of the computer monitor: an initial message, a sample stimulus (a $2 \times 2 \mathrm{~cm}$ green square), and a response cue (a $2 \times 2 \mathrm{~cm}$ purple square). A trial began with the words "click the button when ready." One second after this response, the sample stimulus was presented for a given duration (sample duration). Immediately after termination of the sample stimulus, the response cue appeared and remained visible until the subject responded as described below.

The subjects were told that their job was to terminate the response cue when they estimated that it had been visible for an interval equal to the duration of the sample stimulus. They were instructed to press the mouse button when they estimated that a duration comparable to the sample stimulus duration was about to elapse (start time) and to release it when they estimated that the duration had elapsed (stop time). The stop response terminated the re- sponse cue. The subjects were told that the two responses should always bracket the termination of the sample duration and should create the smallest possible bracket without the sample duration being missed.

Presentation of numeric stimuli. During some trials, three- to five-digit white numbers were presented successively in the center of the sample stimulus, with the first number appearing simultaneously with trial onset. The probability of presenting a number that contained three, four, or five digits was .33 . The mean programmed duration of the three- to five-digit numbers was $1,600 \mathrm{msec}$. The duration of the individual number stimuli varied as a function of the number of digits according to $n$ times $t$, where $n$ is the number of digits and $t$ is a variable duration (mean $=400 \mathrm{msec}$, range $=80$ $720 \mathrm{msec}$ ); however, a number present at the end of a trial was terminated at trial offset, regardless of its programmed duration. Internumber times also varied (mean $=300 \mathrm{msec}$, range $=60-540 \mathrm{msec}$ ). Owing to this programmed variability, the number of numbers appearing during a sample stimulus of a given duration could vary from trial to trial.

Training. The experimenter, who remained in the room during training sessions, orally presented the instructions to each subject and required the subject to verbally state them correctly before the training phase began. There were no number stimuli presented, and no mention was made about the number-reading task during training. Sample durations were randomly selected from a set of whole numbers with a minimum duration of $2 \mathrm{sec}$ and a maximum duration of $23 \mathrm{sec}$. Criterion was met when the subject's start and stop responses bracketed the sample duration on four of five consecutive trials. During training, feedback was presented on the monitor immediately after the stop response. Feedback included the numeric value of the sample duration (in seconds, accurate to two decimal places) and the subject's start and stop times. Feedback remained on the monitor until the subject started the next trial by clicking the mouse button.

Testing. Before each test session, the subjects in each group were told that on some trials, numbers would appear in the center of the green square. They were also told that whether or not numbers appeared in a trial, the objective was to estimate the duration of the green square. The subjects in the passive group were given no further instructions regarding the numbers. The subjects in the active group were told that they should read the numbers aloud as they appeared. Before the first test session, the subjects in this group were shown three numbers written on paper $(142,2,365$, and 57,187) as examples of the numbers they would see in the center of the green square during each session. Next, they practiced reading the numbers aloud, pronouncing each place name-for example, "two thousand three hundred sixty-five." The subjects were told that the computer would record their verbal responses during the experiment; however, this was not the case. Instead, the experimenter listened from an adjacent room, to determine the subjects' compliance with the instruction to read numbers. In the subsequent experiments, the subjects' vocalizations were tape recorded.

For both groups of subjects, test sessions were preceded by 7 warm-up trials. Sample durations on these trials were $t=2.0,3.0$, $4.5,6.7,10.1,15.2$, and $22.8 \mathrm{sec}$ (based on a progression of 1.5t), presented in a random order. Numbers were not presented during warm-up trials, and feedback was provided after each stop response. The experimenter left the room after the warm-up trials; then 56 test trials commenced. Sample durations were the same as those presented during the warm-up trials. Durations were block randomized in 14-trial blocks, with two occurrences of each duration per block. For each duration, numbers were presented during one randomly assigned trial within each block. A different random order of durations was presented in each block.

The subjects participated in three test sessions, approximately $25 \mathrm{~min}$ in length, over a 2-day period. On the 1st day, the subjects were trained; then, following a 5-min break, they underwent the first test session. The second and third test sessions were presented 
on the next calendar day, with a 10-min break between sessions. The subjects reported for experimentation at approximately the same time on each day.

\section{Data Analysis}

For the start-stop procedure, the point of subjective equality (PSE) is based on latency of the start and stop responses from the onset of the response cue. The PSE is taken as (start + stop)/2, termed middle time. The difference limen $(D L)$ is estimated by the standard deviation $(S D)$ of middle time (the measure used in this report) but may also be defined as the difference between stop and start latencies (spread time). Start and stop times were defined as latencies to press and release, respectively, the mouse button, timed from the onset of the response cue.

In all instances in which multiple $t$ tests were employed in post hoc comparisons of a set of means, $\alpha$ levels were adjusted as recommended by Holm (1979). In the figures for all three experiments, signif icant effects are conveyed by presenting confidence intervals $(95 \%)$ that were computed using the method described by Loftus and Masson (1994) for within-subjects designs. The confidence interval was based on the error term for each significant main effect, unless the interaction was significant, in which case it was based on the interaction error term.

\section{Psychophysical Functions}

Results

Group mean middle time for the active and passive groups is plotted for all trial types as a function of sample duration in Figure 1A. Each data point is based on 12 trials. For the three conditions under which the subjects did not perform the number-reading task (no-task trials, active group; filled and empty trials, passive group), mean middle time increased directly with sample duration, approximating veridical time (indicated by the diagonal line). However, under the task condition (active group), estimates departed from veridical time according to a negatively accelerated function.

These impressions were confirmed by analyses of variance (ANOVAs). A group (active vs. passive) $\times$ condition (number presentation vs. no numbers) $\times$ sample duration ANOVA of middle time yielded a significant second-order interaction $\left[F_{\mathrm{S}}(6,108) \geq 25.38, p<.001\right]$. Separate duration $\times$ condition ANOVAs of middle time were performed for each group. For the active group, there were significant effects of duration $[F(6,54)=$ 494.62, $p<.001]$ and condition (task vs. no task) $[F(1,9)=28.30, p<.001]$ and a significant condition $\times$ duration interaction $[F(6,54)=52.13, p<.001]$. Comparison of mean middle times at each sample duration for the task and no-task conditions showed that middle time under task trials was significantly shorter than that under no-task trials for the four longest sample durations ( $t$ tests, $d f=9, p<.05$ ). For the passive group, the only significant effect was that middle time increased with increases in duration $[F(6,54)=1,953.22, p<.001]$.

Trend tests of the relation of middle time to sample duration indicated that functions for the three conditions without number reading were linear, with no significant nonlinear components (proportion of variance accounted for by the linear component was $>.999$ in all cases). Under the task condition for the active group, the func- tion was concave downward; stepwise regression analyses revealed both linear and quadratic components $\left(r^{2}=\right.$ .979 and $>.999$ for linear and added quadratic components, respectively). All slope and intercept values were significantly greater than zero.

For the active group, there were no significant departures from veridical time on no-task trials. On task trials, overestimation was obtained at the shortest duration ( $2 \mathrm{sec}$ ), and underestimation was obtained at the four longest sample durations ( $t$ tests, $d f=9, p<.05$ ). For the passive group, overestimates were found at the shortest sample duration during empty trials ( $t$ tests, $d f=9$, $p<.05)$. For this group, there were no other significant departures from veridical time. Owing to the nonlinear component revealed for the task condition, power functions were fitted to the data from each subject and are presented as group means, plotted in log-log coordinates in Figure 1B. The transformed group functions for all four conditions in Experiment 1 were linear. Group mean slopes, intercepts, and proportions of variance accounted for by the linear trend $\left(r^{2}\right)$ are presented in Table 1, which shows that the slopes were steeper for the conditions in which the subjects did not pronounce numbers (no-task, empty trial, and filled trial conditions) than in the task condition. The inverse relation obtained for the intercepts.

\section{Variability Analysis}

A slope analysis (Ivry \& Corcos, 1993; Ivry \& Hazeltine, 1995) was applied to estimates of variability of the timing process under each experimental condition. A caveat in the use of this analysis is the necessity of adopting a particular representation of the relation between variability in temporal estimates and stimulus magnitude. Variance of estimates may be predicted best by (1) sample duration (for a Poisson process) or (2) the square of the sample duration (for Weber timing). Alternatively, (3) the subject's estimate or (4) the square of the estimate may predict variance best. Finally, (5) analysis of psychophysical data in terms of Weber's law is based on the functional relation between $S D$ s of time estimates and mean time estimates. Ivry and Corcos (1993) and Ivry and Hazeltine (1995) have taken an empirical approach to this issue-evaluating possible models by using linear regression analyses. The slope analysis was then applied to the model providing the best predictive relation between variability of estimates and stimulus magnitude and with nonnegative $y$-intercepts most closely approximating zero. On this basis, Ivry and Corcos conducted a slope analysis based on $S D$ as a function of sample duration, whereas Ivry and Hazeltine used variance as a function of estimate squared, both in accord with Weber's law. In both studies, stimulus durations were less than $1 \mathrm{sec}$.

Regression analyses were conducted for the present data, using the five functional relations described in the previous paragraph. According to the criteria of Ivry and Corcos (1993) and Ivry and Hazeltine (1995), the function relating $S D$ to estimates was the most satisfactory model. Table 2 presents the regression parameters for 


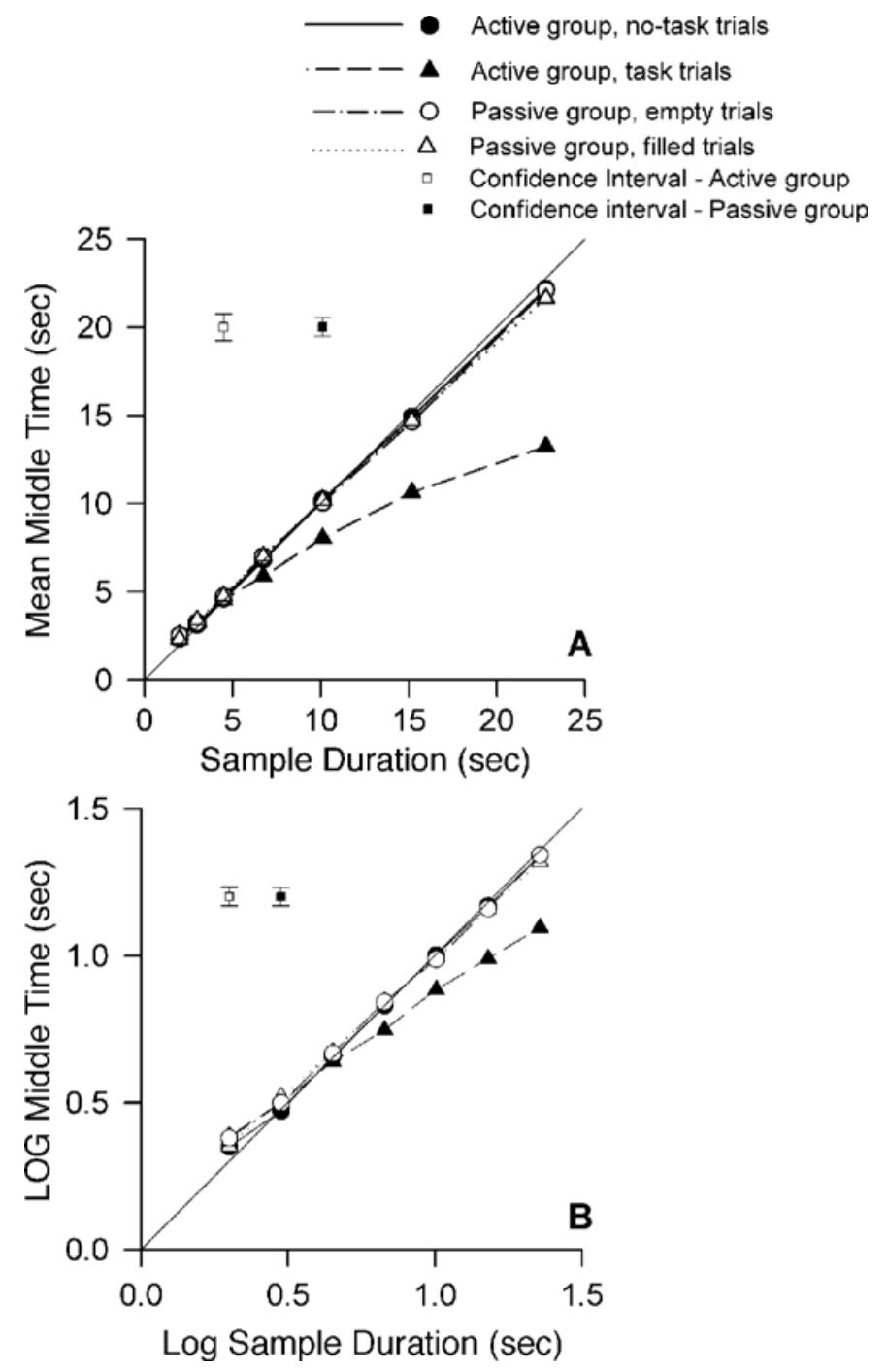

Figure 1. (A) Experiment 1: mean middle time as a function of sample duration on task and no-task trials for the active group and on filled and empty trials for the passive group. The diagonal line indicates veridical time. Confidence intervals for both groups are shown as separate points based on the within-subjects error term for the task $\times$ duration interaction (active group) or for the main effect of duration (passive group). (B) Log middle time as a function of log sample duration on task and no-task trials for the active group and on filled and empty trials for the passive group in Experiment 1. The diagonal line indicates veridical time. Confidence intervals are described above.

this model. For the active group, the group mean slope was greater for task trials than for no-task trials $[F(1,9)=$ $20.01, p<.01]$. For the passive group, there was no significant effect of number presentation on slope $[F(1,9)=$ $2.54, p>.10]$.

\section{Start-Stop and Start-Spread Correlations}

Group mean start-stop and start-spread Pearson correlations for each group and condition were computed. Consistent with the data from human and animal timing preparations, start-stop correlations were positive, and start-spread correlations were primarily negative. In an exception to the latter generalization, the start-spread correlation under the task condition for the active group was positive. Sign tests indicated that both the positive correlation under the task condition and the negative correlation for the no-task condition were reliable $(p<.05)$.

\section{Discussion}

The present data are in accord with those from many other studies that have shown underestimation when a concurrent nontemporal task has been imposed. Al- 
Table 1

Summary of Regression Analyses With Log Sample Duration Predicting Log Time Estimates for All Experiments

\begin{tabular}{|c|c|c|c|c|c|}
\hline Experiment & Group & Condition & Slope & $y$-Intercept & $r^{2}$ \\
\hline \multirow[t]{4}{*}{1} & \multirow[t]{2}{*}{ Active } & $0-0$ (no-task) & 0.96 & 0.04 & .936 \\
\hline & & $1-0$ (task) & $0.68 *$ & $0.18 *$ & $.768^{*}$ \\
\hline & \multirow{2}{*}{ Passive } & Empty trial & 0.92 & 0.08 & .926 \\
\hline & & Filled trial & 0.91 & 0.08 & .893 \\
\hline \multirow[t]{10}{*}{2} & \multirow[t]{2}{*}{$1-0$} & $0-0$ & 0.89 & 0.08 & .851 \\
\hline & & $1-0$ & $0.71 *$ & $0.21 *$ & $.705^{*}$ \\
\hline & \multirow[t]{2}{*}{$0-1$} & $0-0$ & 0.84 & 0.13 & .884 \\
\hline & & $0-1$ & $0.66^{*}$ & $0.38 *$ & $.667 *$ \\
\hline & \multirow[t]{2}{*}{$1-1$} & $0-0$ & 0.93 & 0.05 & .888 \\
\hline & & $1-1$ & $0.73 *$ & $0.27 *$ & $.661^{*}$ \\
\hline & \multirow[t]{4}{*}{ All } & $0-0$ & 0.91 & 0.04 & .799 \\
\hline & & $1-0$ & $0.76^{*}$ & $0.16^{*}$ & .666 \\
\hline & & $0-1$ & 0.86 & $0.21 *$ & .772 \\
\hline & & $1-1$ & $0.78 *$ & $0.20 *$ & .752 \\
\hline \multirow[t]{6}{*}{3} & \multirow[t]{2}{*}{ Reproduction } & $0-0$ & 0.91 & 0.06 & .831 \\
\hline & & $1-0$ & $0.75 *$ & 0.16 & .778 \\
\hline & \multirow[t]{2}{*}{ Estimation } & $0-0$ & 0.91 & 0.08 & .904 \\
\hline & & $1-$ & $0.74 *$ & $0.18^{*}$ & $.808^{*}$ \\
\hline & \multirow[t]{2}{*}{ Production } & $0-0$ & 0.99 & 0.00 & .916 \\
\hline & & -1 & 0.95 & $0.09 *$ & $.797 *$ \\
\hline
\end{tabular}

Note-Entries are group mean values. *Indicates significant difference (related measures $F$ test) in group mean measure between Condition $0-0$ and the given task condition.

though this finding is consistent with an attentional account of task effects, other aspects of the data are not. First, the psychophysical function for the task condition was nonlinear, an outcome that admits of interpretations other than underestimation (Eisler, 1996). Equally im- portant may be the overestimates obtained for the shortest duration and the consistency of the data with previous evidence for Vierordt's law with subjects exposed to concurrent tasks. That is, the psychophysical function has generally been flatter under the task than under the no-task conditions. The results were consistent with the perceptual hypothesis that the temporal judgments under the task and the no-task conditions were mediated by different sensory inputs in the present study.

In accord with the perceptual hypothesis, the slope analysis showed that the growth of variability with increases in estimates differed between the task and the notask conditions. Representation of the psychophysical function in log-log coordinates also yielded support for the perceptual hypothesis. In agreement with prior studies (e.g., Eisler, 1975; Gibbon, 1991; Stevens, 1957), the data were fit well by power relations. The slope of the notask function for the active group was greater than that of the task function; the inverse relation obtained for intercepts. Similar findings have been reported by Brown et al. (1992, Experiment 2). Stevens $(1960,1975)$ noted that the exponent of the power function differs across a wide array of sensory continua. Although the value of an exponent may depend on other factors in addition to sensory modality (see, e.g., Luce \& Krumhansl, 1988; Poulton, 1967, 1968; Stevens, 1975), it is plausible that the variation in exponents under task versus no-task conditions reflects a source of sensory input specific to each condition.

Evidence regarding task effects on memory and threshold mechanisms of the internal clock was sought from start-stop and start-spread correlations, respec-

Table 2

Summary of Regression Analyses With Middle Time Predicting Standard Deviation of Middle Time for All Experiments

\begin{tabular}{|c|c|c|c|c|c|c|}
\hline \multirow[b]{2}{*}{ Experiment } & \multirow[b]{2}{*}{ Group } & \multirow[b]{2}{*}{ Condition } & \multirow[b]{2}{*}{ Slope } & \multirow[b]{2}{*}{$y$-Intercept } & \multicolumn{2}{|c|}{$r^{2}$} \\
\hline & & & & & Linear & Quadratic \\
\hline \multirow[t]{4}{*}{1} & Active & $0-0$ (no-task) & $0.08 *$ & $0.51 *$ & .893 & - \\
\hline & & $1-0$ (task) & $0.18 *$ & 0.33 & .952 & .996 \\
\hline & Passive & Empty trial & $0.07 *$ & $0.42 *$ & .859 & - \\
\hline & & Filled trial & $0.11 *$ & 0.13 & .984 & - \\
\hline \multirow[t]{10}{*}{2} & $1-0$ & $0-0$ & $0.18^{*}$ & 0.34 & .906 & .970 \\
\hline & & $1-0$ & $0.26^{*}$ & -0.01 & .963 & - \\
\hline & $0-1$ & $0-0$ & $0.12 *$ & $0.39 *$ & .938 & - \\
\hline & & $0-1$ & $0.21 *$ & $0.89 *$ & .951 & - \\
\hline & $1-1$ & $0-0$ & $0.13^{*}$ & $0.482 *$ & .944 & - \\
\hline & & $1-1$ & $0.20 *$ & $1.28 *$ & .836 & - \\
\hline & All & $0-0$ & $0.17 *$ & 0.14 & .940 & - \\
\hline & & $1-0$ & $0.25^{*}$ & 0.02 & .909 & - \\
\hline & & $0-1$ & $0.32 *$ & -0.08 & .959 & - \\
\hline & & $1-1$ & $0.23 *$ & 0.42 & .890 & - \\
\hline \multirow[t]{6}{*}{3} & Reproduction & $0-0$ & $0.17 *$ & $0.72 *$ & .917 & - \\
\hline & & $1-0$ & $0.25 *$ & 0.34 & .944 & - \\
\hline & Estimation & $0-0$ & $0.14 *$ & 0.45 & .937 & - \\
\hline & & $1-$ & $0.18 *$ & $0.55 *$ & .970 & _- \\
\hline & Production & $0-0$ & $0.12 *$ & 0.24 & .970 & - \\
\hline & & -1 & $0.28 *$ & -0.28 & .984 & - \\
\hline
\end{tabular}

Note-Entries are group mean values. Slope and intercept values refer to linear regression analyses, and $r^{2}$ values refer to proportion of variance accounted for by linear and added quadratic components, when significant. *Indicates a $y$-intercept or slope value that differs significantly from zero. 
tively. In a replication of previous findings for human and animal subjects (Cheng \& Westwood, 1993; Cheng, Westwood, \& Crystal, 1993; Gibbon \& Church, 1990; Kladopoulos et al., 1998; Rakitin et al., 1998), start-stop correlations were positive under all the conditions of the experiment, and start-spread correlations were negative under three of the four conditions. In an exception to the results in the literature, start-spread correlations were reliably positive under the task condition for the active group. The pattern of high positive start-stop correlations and low but positive start-spread correlations under task conditions implies that the task enhances memory variability relative to threshold variability, according to scalar expectancy theory (SET; Gibbon \& Church, 1990). However, the validity of this analysis in this present study is problematic owing to the nonlinear psychometric function obtained under the task condition. According to SET, perceived discrepancy between elapsed and remembered time on a given trial is a symmetric V-shaped function centered on a perceived discrepancy of zero. Symmetry follows from the assumption that perceived time is linear in real time. Accordingly, the model in its current form does not permit simple inferences regarding the decision process under task conditions.

In the present study, there was relatively little effect of a changing visual display (numbers appearing on the monitor) during the temporal stimuli and no support for a contribution of this variable to the task effect observed for the active group. That the subjects in that group overestimated the shortest duration on task trials does not appear to represent a filled-duration illusion, since this effect was present in the empty trials for the passive group. Factors possibly responsible for differences between the present results and those in studies reporting a filledduration illusion (e.g., Brown, 1995; Thomas \& Brown, 1974) include the properties of the stimuli present during the target interval, such as modality, heterogeneity, and periodicity (Poynter, 1989).

\section{EXPERIMENT 2}

Experiment 1 demonstrated that a concurrent numberreading task was associated with substantial underestimation of longer intervals and a flattening of the psychophysical power function under a reproduction task. In that experiment, the concurrent task was present only during the sample phase of each trial. The purpose of Experiment 2 was to determine whether analogous results would be obtained when the location of the task during the trial was manipulated under the method of reproduction. The task could occur during the sample phase (the 1-0 condition), during the response phase (the $0-1$ condition), during both phases (the $1-1$ condition), or during neither phase (the $0-0$ condition). The analytic procedures in Experiment 1 (with the exception of the analysis based on SET) were used to further evaluate the attentional and perceptual hypotheses regarding task effects.
If task effects include a decrease in the rate of pulse accumulation, as specified by the attentional model, the magnitude of the estimates will vary as a function of task location, at least for the longer durations, for which underestimation was obtained in the previous experiment. When the task is presented during the sample phase only, as in Experiment 1, the subject's experienced duration of the sample will be shortened relative to the no-task condition. Alternatively, when the task occurs during the response phase of the reproduction trial, reproduced durations should exceed those for no-task trials. Under this condition, as the subject executes his/her reproduction of a given sample duration, the concurrent task will reduce the subject's experience of momentary elapsed duration. Interestingly, when the task is present during both the sample and the response phases of a reproduction trial, the aforementioned effects should be canceled out and the magnitude of the temporal estimates should resemble those of the no-task trials. Furthermore, assuming that the rate of pulse accumulation does not differ between the sample and the response phases of the trial under the 1-1 condition, the slope of the function relating reproduced duration to sample duration should approximate a value of 1.0 (Carlson \& Feinberg, 1968). Fortin and Rousseau (1998), using sample durations ranging from 1.6 to $2.4 \mathrm{sec}$, demonstrated task location effects consistent with the foregoing description for the 1-0 and $0-1$ conditions. A short-term memory-processing task was presented during the sample or the reproduction phase of temporal reproduction trials. When memory load was manipulated during the sample phase of the trial, temporal estimates decreased with increases in memory load; the opposite relation was observed when the task was required during the estimation phase.

\section{Method}

\section{Subjects}

Forty undergraduate psychology students ( 25 females and 15 males, 15-55 years of age) participated to satisfy an academic requirement. The subjects were randomly assigned to one of four groups in block random order, with a block size of 4 subjects. No subject failed to complete the experiment.

\section{Apparatus}

The subjects in Groups 1-0, 0-1, and 1-1 were tested using the apparatus described in Experiment 1. The subjects in Group All were tested in a similar experimental setting, using computer hardware and software comparable to those used for the other groups. A tape recorder was used to record the subjects' vocalizations during all the sessions.

\section{Procedure}

With the exceptions noted below, the procedure of this experiment was identical to that in Experiment 1.

Training. The subjects in all the groups were exposed to the training procedures used in Experiment 1, except that instructions were presented on the computer monitor, rather than orally.

Testing. The session and trial structure and the stimulus values were identical to those in Experiment 1, with the exception that the location of the concurrent task within the reproduction trial varied between groups. For the subjects in Groups 1-0, 0-1, and 1-1, the 
task was presented in the sample phase of the trial (the 1-0 condition), the response phase (the $0-1$ condition), or both phases (the 1-1 condition), respectively. For those groups, half of the 56 trials per session were task trials, and half were no-task trials (the $0-0$ condition). The subjects in Group All were presented with all four conditions - the $1-0,0-1,1-1$, and $0-0$ conditions (14 trials for each condition). All the groups were exposed to three test sessions. After the final session, the subjects were asked to describe their strategies for estimating the duration of the sample stimulus under the task and the no-task conditions.

To ensure the subjects' compliance with the instructions to pronounce the numbers presented on the computer monitor, they were accurately informed that their vocal responses would be tape recorded for later review. All the subjects complied at a level of $90 \%$ of the trials or better.

\section{Results}

\section{Psychophysical Functions}

The subjects' estimates (mean middle times) are plotted for all the groups as a function of sample duration in
Figure 2, where the diagonal lines indicate veridical timing. Performance under each of the three task conditions was compared with that in the corresponding no-task condition. The analyses were justified by a significant group $\times$ condition $\times$ duration interaction $[F(12,162)=$ $2.92, p<.01]$ for Groups $1-0,0-1$, and $1-1$ and by a significant condition $\times$ duration interaction for Group All $[F(18,162)=7.90, p<.05]$.

Condition 1-0 versus 0-0. The pattern of results for the $1-0$ condition in comparison with the $0-0$ condition for Group 1-0 and Group All is similar to that for the active group in Experiment 1. No-task functions (the $0-0$ condition) approximated veridical timing; task functions (1-0) were nonlinear and departed from the no-task functions at longer durations.

Condition 0-1 versus 0-0. A different pattern of estimates was obtained for the $0-1$ condition, in which the concurrent task was presented in the response, rather than the sample phase of the trial. Estimates under the

\section{GROUP 1-0}

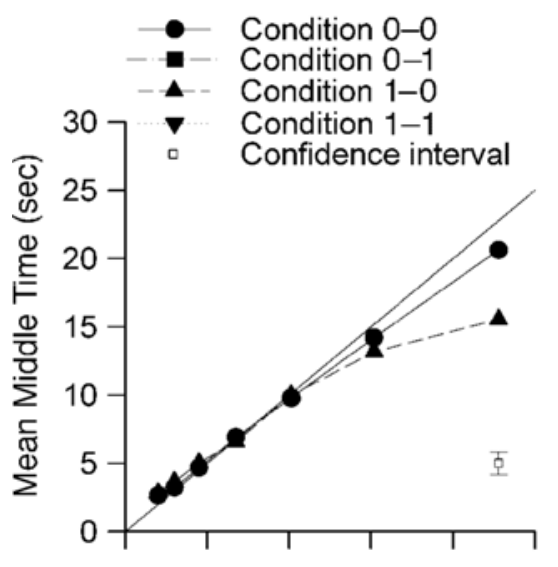

GROUP 1-1

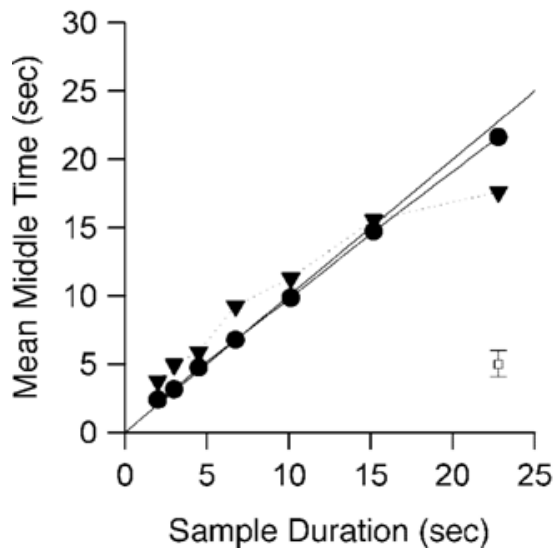

\section{GROUP 0-1}

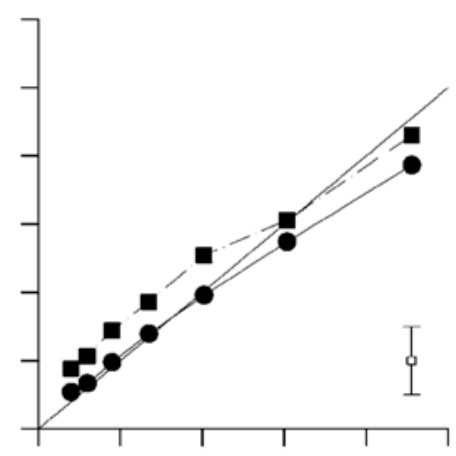

GROUP ALL

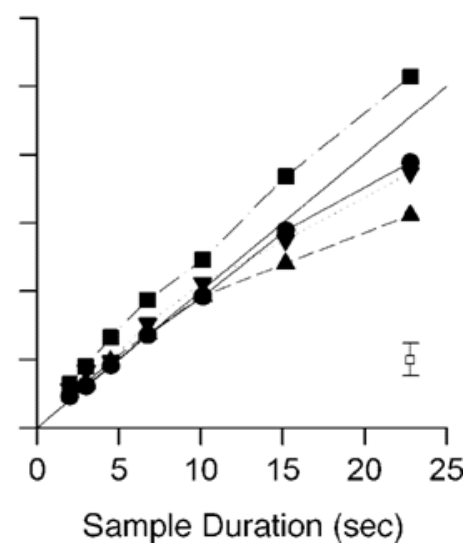

Figure 2. Experiment 2: mean middle time as a function of sample duration for the task and no-task trials for Group 1-0 (upper-left panel), Group 0-1 (upper-right panel), Group 1-1 (lower-left panel), and Group All (lower-right panel). The diagonal line in each panel indicates veridical time. Confidence intervals are shown as separate points based on the within-subjects error term for the task $X$ duration interaction (Groups 1-0, 1-1, and All) or for the main effect of duration (Group 0-1). 
task condition ( $0-1)$ exceeded those in the no-task condition (0-0) for both Group 0-1 and Group All. These differences were all significant for Group All and for the three shortest sample durations for Group 0-1 $(t$ tests, $d f=9, p<.05$ ). Unlike the other task conditions in this experiment, the psychophysical functions for the $0-1$ condition were linear for both Group 0-1 and Group All.

Condition 1-1 versus 0-0. The psychophysical functions for the 1-1 condition were similar to those for the $0-0$ condition in both Group 1-1 and Group All. Estimates differed between the two conditions in only two cases (at the 3.0-sec sample duration for Group All, and at $22.8 \mathrm{sec}$ for Group $1-1 ; t$ tests, $d f=9, p<.05)$. Psychophysical functions under the 1-1 condition were nonlinear (concave downward) for both groups.

When the psychophysical functions were converted to $\log -\log$ coordinates, the resulting functions were linear in all cases (Table 1). The slopes for all the task conditions $(1-0,0-1$, and $1-1)$ were smaller than those for the corresponding no-task condition $(0-0)$ for all the groups (significant for all but the $0-1$ condition of Group All). For the same comparisons, the $y$-intercepts were significantly higher in all cases.

\section{Variability Analysis}

Slope analyses were conducted for the functions relating the $S D$ of middle times to estimates. A linear model provided a good description of the relation: Nine of the 10 functions were linear, and the proportion of variance attributable to a linear component was high (see Table 2). There were no significant negative $y$-intercepts. When the mean function for each task condition was compared with that for its corresponding no-task function, the slope of the task function was significantly greater in all cases.

Self-reported timing strategies. When asked to describe their strategies for estimating the duration of the sample stimulus, all 40 subjects reported some form of chronometric counting under the no-task condition, and only 2 reported an attempt to count seconds (using their fingers) during task conditions. Eleven subjects indicated that they had tried to base their temporal estimates on the number of numbers presented during a trial, and 1 subject reported engaging in a rhythmic movement pattern during the sample stimulus and attempting to reproduce the pattern during the response phase. Most indicated that these strategies were only minimally successful in measuring time.

\section{Discussion}

The observed systematic effect of task location is consistent with the reasoning of Carlson and Feinberg (1968) and indicates that the concurrent task is not merely a nonspecific disrupter of temporal information processing or of timing behavior. As in Experiment 1, the data cannot be entirely explained by reference to a decreased rate of pulse accumulation under task condi- tions. Task functions were largely curvilinear, with the interesting exception of the $0-1$ condition. Furthermore, assuming that the rate of pulse accumulation was invariant between the sample and the response phases of the trial under the $0-0$ and $1-1$ conditions, estimation functions under those conditions should be linear, with slopes approximating 1.0 (Carlson \& Feinberg, 1968). This was not the case, particularly for the 1-1 condition. The slopes of the log-log psychophysical functions and the $D L$ functions suggest that temporal processing differs in a fundamental way under task and no-task conditions. The subjects' self-reports indicated that the sensory bases for their temporal estimates differed under task versus no-task conditions, consistent with the perceptual hypothesis advanced in this article.

\section{EXPERIMENT 3}

In the "evasive art of subjective time measurement" (Zakay, 1990, p. 59), generalizations from different studies are seriously compromised, owing to methodological variables. For that reason, the present study includes a systematic comparison of the effects of the concurrent task used in Experiments 1 and 2 across three conventional psychophysical timing tasks - the methods of reproduction, production, and estimation. The reproduction procedure conformed to the method used in Experiments 1 and 2 (the $1-0$ condition). Under the production procedure, the subjects were required to produce numerically specified temporal intervals, using the start-stop procedure. When present, the nontemporal task occurred as the subjects produced their estimates, as it did under the $0-1$ condition in Experiment 2 . Under the estimation procedure, a visual sample stimulus was presented for a given duration in a method identical to that for the sample phase of the reproduction procedure, after which the subject produced a numeric estimate, using a keyboard. The concurrent task was presented during presentation of the visual stimulus on some trials.

Experiment 3 was performed to assess the generality of the perceptual hypothesis, which anticipates that slopes of $\log -\log$ psychophysical functions and $D L$ functions will vary systematically with presence versus absence of the task, independently of psychophysical method. It also permitted an extension of Stevens and Greenbaum's (1966) regression analysis to timing under a concurrent task procedure. Stevens and Greenbaum argued that psychophysical judgments are influenced by the central tendency of judgment, or regression of judgments toward a central value, an idea they attributed to Hollingworth (1910). Regression has been shown to depend on psychophysicalmethod. Using the psychophysicalcrossmodality matching paradigm, Stevens and Greenbaum presented data indicating that regression occurs when a given stimulus functions as the adjusted dimension, as opposed to when it serves as the standard dimension. The effect was shown for a variety of stimulus modali- 
ties, including duration. Stevens and Greenbaum argued that for duration judgments, the methods of estimation and production might be conceived as instances of crossmodality matching, where duration and numbers function as the stimulus dimensions to be matched. Under the method of estimation, the subject adjusts the number scale, and under the method of production, the subject adjusts the duration of a stimulus. Using stimulus durations ranging from 0.3 to $5.0 \mathrm{sec}$, Stevens and Greenbaum compared performances under the two methods. For each method, numerical values and stimulus values were matched, and the values of the corresponding matches for the two methods were plotted on log-log coordinates. Regression varied inversely with the slope of the matching function, and slope was flatter for the method of estimation than for that of production. The present study extended this investigation to a broader range of durations and to a concurrent task procedure.

As in Experiment 2, to the extent that the concurrent task influences the rate of pulse accumulation, it was anticipated that task effects on the untransformed psychophysical functions would vary with psychophysical method, in accord with the reasoning of Carlson and Feinberg (1968). Consider first the reproduction and estimation procedures. Under both methods, subjects base their estimates on the perceived duration of a visually presented sample stimulus. When the nontemporal task occurs during presentation of the sample stimulus, its effects on perceived duration should be identical under the two procedures. Therefore, apart from differences arising from the differential performance requirements of the two methods (temporal reproduction vs. numeric estimation), psychophysical functions for the methods of reproduction and estimation should appear to be similar and should resemble those for the 1-0 conditions in Experiments 1 and 2. Previous authors have reported that the effects of concurrent tasks are similar under the methods of estimation and reproduction (Brown et al., 1992; Zakay, 1993). To the extent that they are attributable to a change in the rate of pulse accumulation, task effects under the method of production should be symmetric to those observed under the method of reproduction and, by extension, the method of estimation. Accordingly, where task trials were associated with underestimates in Experiment 1 and the 1-0 condition in Experiment 2, overestimates should be obtained under the method of production. Consistent with this reasoning, Brown (1995) and Zakay (1993) have shown inverse relations between temporal judgments under the methods of production and reproduction.

If the effect of the concurrent task is mediated solely by influences on the rate of pulse accumulation, time judgments should be directly proportional to duration under all conditions. However, if the concurrent task introduces sources of sensory input that differ from those under the no-task condition, departures from linearity are to be expected in the untransformed data, as in Experiments 1 and 2 .

\section{Method}

\section{Subjects}

Thirty undergraduate psychology students (17 females and 13 males, 16-47 years of age) participated to satisfy an academic requirement. The subjects were randomly assigned to one of three groups in block random order, with a block size of 6 subjects. The subjects who failed to appear for the experiment, did not meet the training criteria, or failed to follow the instructions were replaced. For the reproduction, production, and estimation groups, 6, 2, and 2 subjects were replaced, respectively.

\section{Apparatus}

The apparatus was the same as that used in Experiment 1.

\section{Procedure}

A different group of subjects was tested under each of three psychophysical procedures - the methods of reproduction, production, and estimation. Stimulus durations for all the groups were integer equivalents of the values used for Experiments 1 and 2-2, 3, $5,7,10,15$, and $23 \mathrm{sec}$. Integer values were used to minimize the perceived difficulty of the temporal production task. Three test sessions of 56 trials were structured identically to those in Experiments 1 and 2. A structured interview followed the last test session.

Reproduction group. The subjects in the reproduction group were exposed to the same general methodology as that used for the active group and Group 1-0 in Experiments 1 and 2, respectively. Procedural changes were made to the training procedure, which was automated for the present experiment, and to the stimulus durations (described above).

During the training session, instructions were presented on the computer monitor, as in Experiment 2; however, a computer program, rather than the experimenter, monitored the subject's performance and terminated the session when a performance criterion was achieved or after 100 trials. To complete training, a subject was required to produce four of five consecutive estimates in which the response bracketed the target duration and jointly met the criteria for start, stop, and middle times. The start response requirement was that it should occur no later than $95.5 \%$ of the target time; the stop response was required to occur no sooner than $100.5 \%$ and no later than $1000 \%$ of the target duration; the middle time was required to fall within $20 \%$ of the target duration. Liberal criteria were chosen in order to provide instructions that would place only minor constraints on the timing of responses. Three subjects failed to meet the training criterion. Although the loss of subjects in this group was higher than that for the production and estimation groups, performance was similar to that observed in Experiments 1 and 2 under nearly identical conditions.

Production group. The method of production was programmed similarly to the method of reproduction, except that only the response cue was presented on each trial. After the subject initiated a trial by clicking the mouse button, a message indicating the target time was shown at the top of the computer monitor. Below the message, centered on the monitor, was a purple or a green square (balanced across subjects), as in the method of reproduction. The subject was instructed in using the start-stop procedure to produce a temporal estimate. Training was accomplished using the automated procedure described for the reproduction group, except that the method of production was used. All the subjects completed training.

Estimation group. The subjects initiated each estimation trial by clicking the mouse button, after which a green or a purple square (balanced across subjects) was presented on the computer monitor for the target duration. When the stimulus ended, the following instruction was presented: "TYPE YOUR ESTIMATE IN SECONDS AND PRESS THE ENTER KEY."

An automated training procedure was used, and the subjects were required to produce four of five consecutive estimates that were 


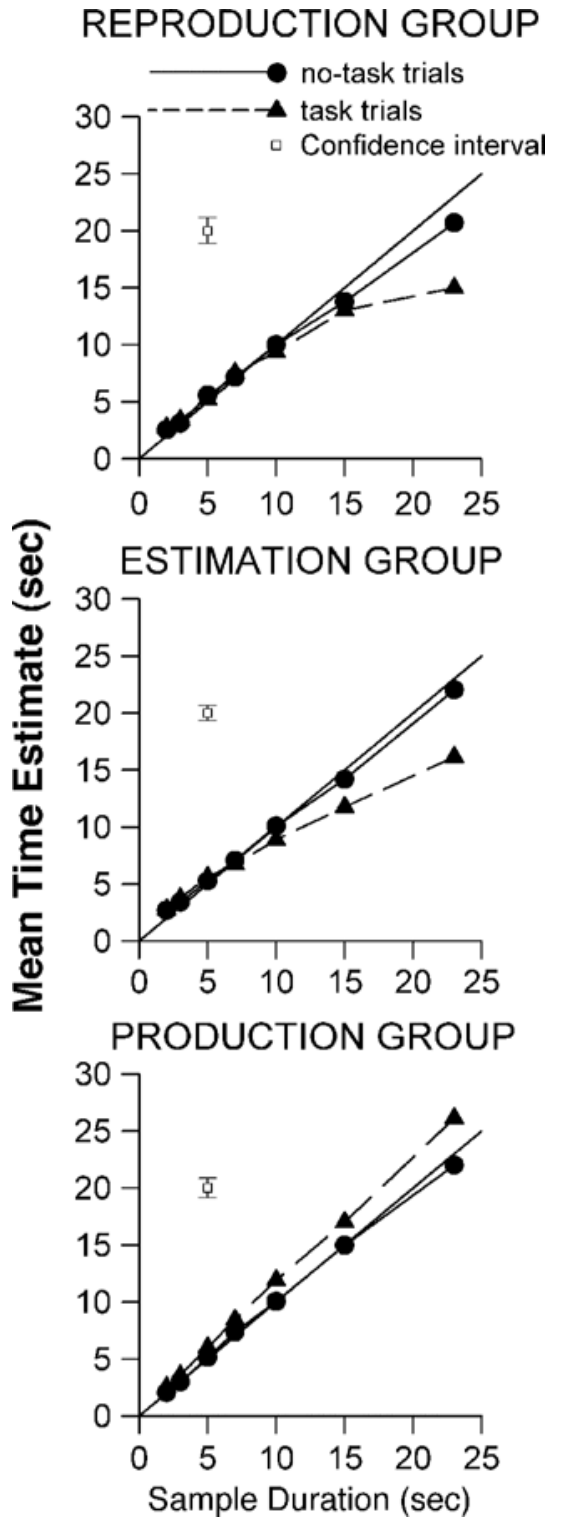

Figure 3. Experiment 3: mean time estimate as a function of sample duration for the reproduction (upper panel), estimation (middle panel), and production (lower panel) groups. The diagonal line in each panel indicates veridical time. Confidence intervals are shown as separate points based on the within-subjects error term for the task $\times$ duration interaction.

within $20 \%$ of the target duration (analogous to the criterion for middle time for the reproduction and production groups). All the subjects met the training criterion.

\section{Results}

\section{Psychophysical Functions}

Temporal estimates as a function of sample duration are plotted on separate axes for each group in Figure 3. Performance on no-task trials was similar under the three psychophysical methods; however, group differences were apparent on task trials. As was anticipated for those trials, psychophysicalfunctions for the production method differed from those for the estimation and reproduction methods. The latter were similar to each other and to those for the 1-0 conditions in Experiments 1 and 2.

An ANOVA of judgments, with group, condition (task vs. no task), and duration as factors, revealed a significant group $\times$ condition interaction $[F(2,27)=9.76, p<$ $.001]$ and a significant group $\times$ condition $\times$ duration interaction $[F(12,162)=12.73, p<.001]$. (Significant effects were also found for group, duration, group $\times \mathrm{du}-$ ration, and condition $\times$ duration.) Separate ANOVAs for each group indicated significant condition $\times$ duration interactions for all the groups. Task functions were negatively accelerated for the reproduction and estimation groups and were linear for the production group. Furthermore, for the estimation and reproduction groups, task functions were lower than no-task functions at the longer sample durations, whereas for the production group, the task function was higher than the no-task function. Significant differences between task and notask trials were obtained for the 23 -sec sample duration for the reproduction group, for the 10-, 15-, and 23-sec durations for the estimation group, and for the 2-, 3-, 5-, $10-$, and 23-sec durations for the production group ( $t$ tests, $d f=9, p<.05$, for each group).

The effect of psychophysical method was confined to the task trials, as was indicated by two-way ANOVAs with group and duration as factors, conducted separately for task and no-task trials. For task trials, there were significant effects of group $[F(2,27)=8.30, p<.01]$ and duration $[F(6,162)=397.72, p<.001]$ and a significant group $\times$ duration interaction $[F(12,162)=19.01$, $p<.001]$. Only the effect of duration was significant for the no-task data $[F(6,162)=1,196.30, p<.001]$.

When the psychophysical functions were converted to $\log -\log$ coordinates, the resulting functions were linear in all cases (Table 1). The slopes for all the task conditions were lower than those for the corresponding no-task condition for all the psychophysical methods, although the difference was not significant for the method of production. For the same comparisons, the $y$-intercepts were higher in all cases, a significant effect for the methods of estimation and production.

\section{Variability Analysis}

$S D$ increased as a linear function of mean middle time for task and no-task trials under each of the three procedures (Table 2). There were no significant negative $y$-intercepts. As in Experiments 1 and 2, the slopes of the $S D$ functions were steeper for the task than for the no-task functions. A group $\times$ condition (task vs. no task) ANOVA showed a significant effect of condition $[F(1,27)=9.49$, $p<.01]$, no effect for group, and no group $\times$ condition interaction $[F(2,27)=1.8$ and 1.52 , respectively $]$.

\section{Analysis of Regression Effects}

To assess the possibility that task-trial performance under the three psychophysical methods was differen- 
tially influenced by regression, the data were analyzed in the manner of Stevens and Greenbaum (1966). Following $\log$ transforms of both axes, numerical values and stimulus values were matched. The resulting functions were linear in all cases $\left(r^{2}\right.$ values were $.978, .984$, and .968 for the estimation, production, and reproduction groups, respectively). In accord with the findings of Stevens and Greenbaum, the slope for the estimation function (0.74) was lower than that for the production function (1.05). Owing to the methodological similarity between the estimation and the reproduction procedures, the analysis was also applied to the data for the latter, yielding a measure comparable to that for the estimation group $($ slope $=0.75)$.

\section{Self-Reported Timing Strategies}

The results of the structured interview were similar to those in Experiment 2. The subjects reported counting under the no-task condition and difficulty in monitoring duration under the task condition. Nine subjects reported trying to base their estimates on the number of numbers that had appeared during the trial, and 3 tried to count seconds with their fingers. Two subjects reported trying to count as they pronounced the numbers, and 1 tried to count in between number presentations.

\section{Discussion}

The results in Experiment 3 are in accord with those in Experiments 1 and 2 in their support of the perceptual hypothesis. The slopes of the functions relating $S D \mathrm{~s}$ to estimates were higher under task than under no-task conditions, whereas the slopes of the log-log psychophysical functions were lower under task conditions. Although these effects were independent of psychophysicalmethod, the method variable did interact with task condition in determining the shape of the untransformed psychophysical functions. As was anticipated, the relations of task to no-task functions for the reproduction and the estimation groups were similar to one another-the task was associated with lower estimates at the longer durations, and the psychophysical functions were curvilinear (Figure 3). On the other hand, the subjects in the production group produced a linear task function and showed higher estimates under task than under no-task conditions at most sample durations. In contrast to the findings of Rakitin et al. (1998), who used a similar concurrent task, the present study indicates that performance under the method of production is profoundly influenced by a concurrent number-reading task. Conceivably, presentation of feedback following each estimate by Rakitin et al. mitigated task effects in their study.

The observed differences in the direction of task effects across methods were in line with predictions based on the reasoning of Carlson and Feinberg (1968); however, in a departure from that account, a symmetry of task effects under production and estimation procedures was not obtained. An analysis of the psychophysical functions, using the method of Stevens and Greenbaum
(1966), suggested that the lack of symmetry might be understood in terms of differential regression effects under the two procedures. In accord with data reported by Stevens and Greenbaum, regression was greater for the method of estimation than for that of production. It is noteworthy that, in comparison with their data, the slopes obtained in the present analysis were somewhat low. Because Stevens and Greenbaum described no attempts to discourage subjects from engaging in chronometric counting, it may be more reasonable to compare their data with those from the no-task conditions for the production and estimation groups. When the temporal intervals were signaled by a visual stimulus, Stevens and Greenbaum reported slopes of 0.93 and 1.16 for estimation and production, respectively; comparable values under the no-task condition in the present study were similar at 0.91 and 1.01. In summary, the foregoing analyses indicate that method-based regression effects exerted an influence upon scaling under the present psychophysical procedures.

\section{GENERAL DISCUSSION}

In three experiments, a concurrent number-reading task produced consistent and marked effects on temporal judgments. To a great extent, the results conform to those in the empirical literature, which indicate that concurrent tasks are associated with a decrease in reported duration under prospective-timing procedures. Accordingly, they are consistent with the widely accepted interpretation of concurrent task effects based on attentional resource allocation models. However, the consistently negatively accelerated psychophysical functions under the methods of reproduction and estimation indicate that attentional effects, mediated by changes in the rate of pulse accumulation, do not adequately account for the present pattern of results.

In an alternative interpretation of the present findings, the perceptual hypothesis argues that different sources of sensory input control judgments under task and no-task conditions. The subjects reported that their judgments under the no-task condition were based on chronometric counting but that counting was difficult, if not impossible, under the task condition. Thus, task and no-task intervals differed considerably with respect to the availability of sources of stimulation on which time judgments could be based. In support of the perceptual hypothesis, the functions relating $D L$ to time judgments were steeper for task than for no-task conditions in 10 out of 10 independent cases $[p<.001$, sign test; compare the $0-0$ condition with the corresponding task condition(s) for each experimental group, except the passive group; see Table 2]. Previous findings that the value of the minimal Weber fraction differs across sensory modalities (e.g., Woodworth $\&$ Schlosberg, 1954) support the interpretation of the present effects in terms of differing sensory input.

Additional evidence consistent with the perceptual hypothesis was obtained by plotting the psychophysical 
functions on $\log$ - $\log$ coordinates. As is shown in Table 1, all 20 transformed group functions from Experiments 1, 2 , and 3 were linear, and the slopes for the no-task condition were steeper than those for the task condition in all cases (significant in 8 out of 10 comparisons), regardless of psychophysical method. Exponents for the notask condition averaged .92, close to the values reported by Eisler $(1975,1976)$, but different from the more recently reported exponent of 1.0 (Allan, 1983; Allan \& Gibbon, 1991). For the no-task conditions, the proportion of variance accounted for by the power function was reasonably high and was higher in all the experiments than that for the task condition (Table 1). This difference is consistent with the well-documented finding that the variability of time judgments under task conditions is greater than that under no-task conditions (Brown, 1997).

The data from the task and no-task conditions supporting the perceptual hypothesis may also be interpreted in terms of other variables (Poulton, 1968), or could represent lowered sensitivity to duration under task conditions (Brown et al., 1992; Catania, 1970; Staddon \& Higa, 1999). Teghtsoonian (1971) observed that there was a systematic negative correlation between the exponent of the power function and the ratio of the largest to the smallest stimulus intensity employed in a variety of judgment tasks. This relation suggested that stimulus dimensions that differ in dynamic range map onto similar sensory ranges. Consistent with this idea was the observation that the Weber fraction expressed in terms of stimulus units varied inversely with the exponent across dimensions in a manner that led to invariance in the subjective Weber fraction. The negative relation of exponent and Weber fraction obtained across task and no-task conditions in the present study is consistent with this perceptual theory. It also implies that the dynamic range of durations under task conditions is greater than that under no-task conditions.

According to the perceptual hypothesis, the reduction in time judgments often observed under task conditions with prospective estimation procedures are improperly construed as underestimation or subjective shortening; rather, they represent the outcome of a parameter adjustment in a scaling rule. The extent to which an attentiondriven decrease in the rate of pulse accumulation mediates time judgments under task conditions remains an issue to be addressed (see, e.g., Killeen \& Taylor, 2000), but the present results call into question an exclusive reliance on this account of task effects in human timing.

\section{REFERENCES}

Allan, L. G. (1983). Magnitude estimation of temporal intervals. Perception \& Psychophysics, 33, 29-42.

Allan, L. G., \& GibBon, J. (1991). Human bisection at the geometric mean. Learning \& Motivation, 22, 39-58.

BLOCK, R. A. (1992). Prospective and retrospective duration judgement: The role of information processing and memory. In F. Macar, V. Pouthas, \& W. J. Friedman (Eds.), Time, action, and cognition: Towards bridging the gap (pp. 141-152). Dordrecht: Kluwer.

BLOCK, R. A., \& ZAKAY, D. (1996). Models of psychological time re- visited. In H. Helfrich (Ed.), Time and mind (pp. 171-195). Seattle: Hogrefe \& Huber.

Brown, S. W. (1995). Time, change, and motion: The effects of stimulus movement on temporal perception. Perception \& Psychophysics, 57, 105-116.

BROWN, S. W. (1997). Attentional resources in timing: Interference effects in concurrent temporal and nontemporal working memory tasks. Perception \& Psychophysics, 59, 1118-1140.

Brown, S. W., StubBs, D. A., \& West, A. N. (1992). Attention, multiple timing, and psychophysical scaling of temporal judgements. In F. Macar, V. Pouthas, \& W. J. Friedman (Eds.), Time, action, and cognition: Towards bridging the gap (pp. 129-140). Dordrecht: Kluwer.

CARLSON, V. R., \& FEINBERG, I. (1968). Individual variations in time judgement and the concept of an internal clock. Journal of Experimental Psychology, 77, 631-640.

CATANiA, A. C. (1970). Reinforcement schedules and psychophysical judgments: A study of some temporal properties of behavior. In W. N. Schoenfeld (Ed.), The theory of reinforcement schedules (pp. 1-42). New York: Appleton-Century-Crofts.

Cheng, K., \& Westwood, R. (1993). Analysis of single trials in pigeons' timing performance. Journal of Experimental Psychology: Animal Behavior Processes, 19, 56-67.

Cheng, K., Westwood, R., \& Crystal, J. D. (1993). Memory variance in the peak procedure of timing in pigeons. Journal of Experimental Psychology: Animal Behavior Processes, 19, 68-76.

EISLER, H. (1975). Subjective duration and psychophysics. Psychological Review, 82, 429-450.

EISLER, H. (1976). Experiments on subjective duration 1868-1975: A collection of power function exponents. Psychological Bulletin, 83, 1154-1171.

EISLER, H. (1996). Time perception from a psychophysicist's perspective. In H. Helfrich (Ed.), Time and mind (pp. 65-86). Seattle: Hogrefe \& Huber.

Fetterman, J. G., \& Killeen, P. R. (1990). A componential analysis of pacemaker-counter timing systems. Journal of Experimental Psychology: Human Perception \& Performance, 16, 766-780.

Fortin, C., \& Rousseau, R. (1998). Interference from short-term memory processing on encoding and reproducing brief durations. Psychological Research, 61, 269-276.

Fortin, C., Rousseau, R., Bourque, P., \& Kirouac, E. (1993). Time estimation and concurrent nontemporal processing: Specific interference from short-term-memory demands. Perception \& Psychophysics, 53, 536-548.

FRANKENHAEUSE R,M. (1959). Estimation of time: An experimental study. Stockholm: Almquist \& Wiksell.

GibBon, J. (1991). Origins of scalar timing. Learning \& Motivation, 22, 3-38.

Gibbon, J., \& Church, R. M. (1990). Representation of time. Cognition, 37, 23-54

Grondin, S., Meilleur-Wells, G., \& Lachance, R. (1999). When to start explicit counting in a time-intervals discrimination task: A critical point in the timing process of humans. Journal of Experimental Psychology: Human Perception \& Performance, 25, 993-1004.

Hicks, R. E., Miller, G. W., Gaes, G., \& Bierman, K. (1977). Concurrent processing demands and the experience of time-in-passing. American Journal of Psychology, 90, 431-446.

HoLm, S. (1979). A simple sequentially rejective multiple test procedure. Scandinavian Journal of Statistics, 6, 65-70.

IVRY, R. [B.], \& CoRCos, D. M. (1993). Slicing the variability pie: Component analysis of coordination and motor dysfunction. In K. M. Newell \& D. M. Corcos (Eds.), Variability and motor control (pp. 415-447). Champaign, IL: Human Kinetics.

IVRY, R. B., \& HAZELtine, R. E. (1995). Perception and production of temporal intervals across a range of durations: Evidence for a common timing mechanism. Journal of Experimental Psychology: Human Perception \& Performance, 21, 3-18.

KILleEN, P. R., \& TAYLOR, T. J. (2000). How the propagation of error through stochastic counters affects time discrimination and other psychophysical judgments. Psychological Review, 107, 430-459.

Killeen, P. R., \& Weiss, N. A. (1987). Optimal timing and the Weber function. Psychological Review, 94, 455-468. 
Kladopoulos, C. N., Brown, B. L., Hemmes, N. S., \& Cabeza de VACA, S. (1998). The start-stop procedure: Estimation of temporal intervals by human subjects. Perception \& Psychophysics, 60, 438-450.

Kladopoulos, C. N., Hemmes, N. S., \& Brown, B. L. (1997, April). The start-stop procedure: Effects of a concurrent nontemporal task. Paper presented at the annual meeting of the Eastern Psychological Association, Washington, DC.

LEJEUNE, H. (1998). Switching or gating? The attentional challenge in cognitive models of psychological time. Behavioural Processes, 44, 127-145.

LofTus, G. R., \& MAsson, M. E. J. (1994). Using confidence intervals in within-subject designs. Psychonomic Bulletin \& Review, 1, 476490.

LuCE, R. D., \& KRumhansL, C. L. (1988). Measurement, scaling, and psychophysics. In R. C. Atkinson, R. J. Herrnstein, G. Lindzey, \& R. D. Luce (Eds.), Stevens' handbook of experimental psychology: Vol. 1. Perception and motivation (pp. 3-74). New York: Wiley.

MaCAR, F., Grondin, S., \& CASINI, L. (1994). Controlled attention sharing influences time estimation. Memory \& Cognition, 22, 673-686.

Poulton, E. C. (1967). Population norms of top sensory magnitudes and S. S. Stevens' exponents. Perception \& Psychophysics, 2, 312-316.

Poulton, E. C. (1968). The new psychophysics: Six models of magnitude estimation. Psychological Bulletin, 69, 1-19.

POYNTER, W. D. (1989). Judging the duration of time intervals: A process of remembering segments of experience. In I. Levin \& D. Zakay (Eds.), Time and human cognition: A life span perspective (pp. 305331). Amsterdam: Elsevier, North-Holland.

Poynter, W. D., \& Homa, D. (1983). Duration judgment and the experience of change. Perception \& Psychophysics, 33, 548-560.

Rakitin, B. C., Gibbon, J., Penney, T. B., Malapani, C., Hinton, S. C., \& MECK, W. H. (1998). Scalar expectancy theory and peak-interval timing in humans. Journal of Experimental Psychology: Animal Behavior Processes, 24, 15-33.

ROBERTS, S. (1981). Isolation of an internal clock. Journal of Experimental Psychology: Animal Behavior Processes, 7, 242-268.

SAwYer, T. F., MeYers, P. J., \& Huser, S. J. (1994). Contrasting task demands alter the perceived duration of brief time intervals. Perception \& Psychophysics, 56, 649-657.

StAdDON, J. E. R., \& HigA, J. J. (1999). Time and memory: Towards a pacemaker-free theory of interval timing. Journal of the Experimental Analysis of Behavior, 71, 215-251.
Ste Vens, S. S. (1957). On the psychophysical law. Psychological Review, 64, 153-181.

STE VENS, S. S. (1960). The psychophysics of sensory function. American Scientist, 48, 226-253.

STE VENS, S. S. (1975). The psychophysical law. In G. Stevens (Ed.), Psychophysics: Introduction to its perceptual, neural, and social prospects (pp. 1-36). New York: Wiley.

Ste vens, S. S., \& Greenbaum, H. B. (1966). Regression effect in psychophysical judgment. Perception \& Psychophysics, 1, 439-446.

Teghtsoonian, R. (1971). On the exponents in Stevens' law and the constant in Ekman's law. Psychological Review, 78, 71-80.

Thomas, E. A. C., \& Brown, I., JR. (1974). Time perception and the filled-duration illusion. Perception \& Psychophysics, 16, 449-458.

Thomas, E. A. C., \& Weaver, W. B. (1975). Cognitive processing and time perception. Perception \& Psychophysics, 17, 363-367.

Treisman, M. (1963). Temporal discrimination and the indifference interval: Implications for a model of the "internal clock." Psychological Monographs, 77(Whole No. 576).

VROON, P. A. (1970). Effects of presented and processed information on duration experience. Acta Psychologica, 34, 115-121.

Wallace, M., \& Rabin, A. (1960). Temporal experience. Psychological Bulletin, 57, 213-236.

Wearden, J. H., Denovan, L., Fakhri, M., \& Haworth, R. (1997). Scalar timing in temporal generalization in humans with longer stimulus durations. Journal of Experimental Psychology: Animal Behavior Processes, 23, 502-511.

Woodrow, H. (1951). Time perception. In S. S. Stevens (Ed.), Handbook of experimental psychology (pp. 1224-1236). New York: Wiley.

WoOdWORTH, R. S., \& SCHLOSBERG, H. (1954). Experimental psychology. New York: Holt.

ZAKAY, D. (1990). The evasive art of subjective time measurement: Some methodological dilemmas. In R. A. Block (Ed.), Cognitive models of psychological time (pp. 59-84). Hillsdale, NJ: Erlbaum.

ZAKAY, D. (1993). Time estimation methods-do they influence prospective duration estimates? Perception, 22, 91-101.

ZAKAY, D. (1998). Attention allocation policy influences prospective timing. Psychonomic Bulletin \& Review, 5, 114-118.

(Manuscript received September 20, 2001; revision accepted for publication August 5, 2003.) 